\title{
Linkage disequilibria between mtDNA haplotypes and chromosomal arrangements in a natural population of Drosophila subobscura
}

\author{
P Oliver ${ }^{1}$, JA Castro ${ }^{1}$, A Picornell ${ }^{1}$, MM Ramon ${ }^{1}$, E Solé ${ }^{2}$, J Balanyà ${ }^{2}$, L Serra ${ }^{2}$, A Latorre ${ }^{3}$ and \\ A Moya ${ }^{3}$ \\ ${ }^{1}$ Laboratori de Genètica, Departament de Biologia, Universitat de les Illes Balears, Palma de Mallorca, Balears, Spain; ${ }^{2}$ Departament \\ de Genètica, Universitat de Barcelona, Barcelona, Spain; ${ }^{3}$ Institut Cavanilles de Biodiversitat i Biologia Evolutiva i Departament de \\ Genètica, Universitat de València, Burjassot, València, Spain
}

\begin{abstract}
The association between mtDNA haplotypes and chromosomal arrangements in a natural population of Drosophila subobscura from Calvia (Balearic Islands, Spain) was studied in order to search for linkage disequilibria, in an attempt to explain the populational dynamics of the mtDNA haplotypes of this species in nature. The presence of Wolbachia was not detected. Two main haplotypes (I and II) were
\end{abstract}

found, as well as a series of less common ones. The Tajima $D$-test seemed to indicate some kind of seasonal population bottleneck. An analysis of linkage disequilibrium and factorial analysis of correspondences detected an association between haplotype I and the $\mathrm{J}_{\mathrm{ST}}$ inversion and haplotype II and the $\mathrm{J}_{1}$ inversion.

Heredity (2002) 89, 133-138. doi:10.1038/sj.hdy.6800116

Keywords: linkage disequilibria; mtDNA; haplotypes; chromosomal arrangements; Drosophila subobscura

\section{Introduction}

Drosophila subobscura is a Palaearctic species of the obscura subgroup of Drosophila, and is distributed all over Europe (except central and northern Scandinavia), the Middle East, northern Africa and the Atlantic islands of the Azores, Madeira, and the Canaries. In addition, it has recently colonized the American continent (Ayala et al, 1989, and references therein).

A consistent observation in studies of mitochondrial DNA evolution in Old and New World populations of this species for more than 15 years is the high prevalence of two widespread and almost equally frequent haplotypes (I and II) and the sporadic appearance of rare ones at a low frequency (Latorre et al, 1986; García-Martínez et al, 1998; Castro et al, 1999). The exception is the Canary Islands, where an endemic haplotype is the most frequent on some islands (Pinto et al, 1997). Moya et al (1993) studied the degree of differentiation at the nucleotide level for the two main haplotypes. Only three differences were found and they proved to be silent changes at the protein level; one of which corresponded to the HaeIII restriction site that distinguishes both haplotypes which is located in the ND5 gene.

It is still an unresolved question which evolutionary forces account for the coexistence of the main haplotypes I and II in nature. In the literature, studies concerning the population genetic dynamics of mtDNA have often,

Correspondence: Dr JA Castro, Laboratori de Genètica, Departament de Biologia, Facultat de Ciències, Edifici Guillem Colom, Universitat de les Illes Balears, Campus de la UIB, 07071 Palma de Mallorca (Balears), Spain. E-mail: jose.castro@uib.es

Received 30 May 2001; accepted 22 April 2002 although not always, shown the rejection of neutral patterns, mainly in humans and their associated commensal taxa (flies and mice) (see Gerber et al (2001) for a recent revision). In this way, Nachman et al (1994) in Mus species and Nachman et al (1996) in humans and chimpanzees, and Kennedy and Nachman (1998) in rats, have shown that mtDNA nucleotide sequences deviate from a neutral model, and this has been interpreted as being caused by a large class of mildly deleterious mutations. Taking into account the changes in the mtDNA haplotype frequencies in experimental populations and fitness estimates in Drosophila, MacRae and Anderson (1988, 1990) and Jenkins et al (1996) suggested that mtDNA evolves in a non-neutral fashion in D. pseudoobscura. Fos et al (1990) discuss the importance of positive natural selection in $D$. subobscura mtDNA haplotypes, but also consider the effect of the nuclear genetic background to be very important and, in conjunction with random drift, this dictates the final outcome where there are competing haplotypes. Kilpatrick and Rand (1995) failed to obtain evidence of selection in competition experiments between mitochondrial haplotypes in D. melanogaster, but their results were interpreted as being caused by a temporary hitchhiking of nuclear background on mtDNA. In an extensive report, Rand and Kann (1998) enlarged the study to non-commensal species (fishes, birds, bears) and they found evidence for both neutral and nonneutral patterns of diversity. In an experiment with the D. subobscura haplotypes I and II, García-Martínez et al (1998) observed the fixation of haplotype II over haplotype I in population cages. They rejected random drift as the force responsible for the observed changes in haplotype frequencies in these population cages, which contrasted with the stability found in nature. They also pointed out 
that the shift in mtDNA haplotype frequencies would not necessarily be caused by selection acting on mtDNA variants themselves, but also possibly by temporary hitchhiking of mtDNA haplotypes with selected nuclear genetic variation, or a functional co-adaptation between the nuclear and mitochondrial genomes. Castro et al (1999) studied the genetic structure of $D$. subobscura from different locations of Majorca and Minorca (Balearic Islands, Spain) by means of allozymes and mitochondrial DNA. In a cytonuclear disequilibrium analyses, they showed the difficulty of detecting significant cytonuclear associations in natural populations because these turned out mainly to be transient.

Chromosomal polymorphism for inversions of $D$. subobscura has been extensively studied both in Europe and in the recently colonized areas of North and South America (Krimbas, 1993; Prevosti et al, 1988). The geographical pattern of latitudinal variation for most of the gene arrangements is the same in these three areas, which shows the adaptive nature of this polymorphism, which is mainly mediated by temperature. This adaptation to climatic conditions has also been detected by temporal changes in inversion frequencies (Orengo and Prevosti, 1996). Therefore, it is interesting to consider the possible relation between the mtDNA haplotypes and chromosomal arrangements in terms of hitchhiking, because if an association exists and is selectively maintained, it could help to understand the dynamics of the different mtDNA haplotypes in nature.

For these reasons, the main purpose of the current study was to test any association between inversion polymorphism and the mtDNA haplotypes in a natural population of D. subobscura.

\section{Materials and methods}

Two hundred and one isofemale lines of D. subobscura were sampled from a pine forest near Calvia (Island of Majorca) in the spring of 1997. The flies were collected with conventional traps using fermented bananas. Once in the laboratory, the females were placed individually in vials with food and kept in an incubator at $19^{\circ} \mathrm{C}$. When the $F_{1}$ larvae appeared, the offspring were used to determine the maternal mitochondrial haplotypes. Males from the $F_{1}$ larvae were chosen to analyse the chromosomal arrangements. In this way, from each isofemale line, information was obtained about the mtDNA haplotype and the inversions of one chromosome set.

\section{Extraction and digestion of mtDNA}

An enriched fraction of mtDNA was obtained using the method described by Latorre et al (1986). This fraction was digested with five restriction enzymes. Three of the enzymes (EcoRI, EcoRV and HindIII) recognize sequences of $6 \mathrm{bp}$, whereas the other two (HpaII and HaeIII) sequences of $4 \mathrm{bp}$. These enzymes were selected for their ability to detect mtDNA polymorphisms (Castro et al, 1999, and references therein). The fragments obtained by digestion were separated on horizontal 1-2\% agarose gels. To determine fragment size, DNA molecular weight markers (II, IV and VI) (Roche) were used as size standards. After electrophoresis, gels were stained with 0.1 $\mu \mathrm{g}$ ethidium bromide $\mathrm{mL}^{-1}$ and visualized with a $260 \mathrm{~nm}$ UV light transilluminator. A mtDNA restriction map was obtained by means of all possible single and double digestions of the mtDNA. The different restriction patterns obtained, using a given enzyme and the haplotypes, were named according to the notation of Latorre et al (1986, 1992) and Castro et al (1999).

\section{Inversion polymorphism analysis}

A male of each isofemale line was individually crossed with females from the laboratory strain chcu, which is homokaryotypic for all the chromosomes $\left(\mathrm{A}_{\mathrm{ST}}, \mathrm{J}_{\mathrm{ST}}, \mathrm{E}_{\mathrm{ST}}\right.$, $\mathrm{O}_{3+4}, \mathrm{U}_{\mathrm{ST}}$ ). The polytene chromosomes of one third instar female larvae were stained and squashed in lacto-acetoorcein solution.

\section{Statistical analyses}

Tajima's D-test (Tajima, 1989) was used to test for any departure from neutrality for the mtDNA haplotype distribution in the population. The rationale of the test is that in a panmictic population, under the neutral mutation model, no difference would be expected between the average number of nucleotide differences (ie nucleotide heterozygosity) and the number of segregating sites, when the latter is adjusted by dividing by $\sum_{i=1}^{i=n-1} 1 / i$, where $i$ is the $i$ th sequence and $n$ the sample size.

To test for linkage disequilibrium between the chromosomal arrangements and the mtDNA haplotypes, the $D$ and $D^{\prime}$ (Lewontin, 1964) parameters were used.

$$
D_{i j}=p_{i j}-p_{i} p_{j}
$$

where $D_{i j}$ is the disequilibrium value between the $i$ arrangement and the $j$ haplotype; $p_{i j}$ is the frequency of the combination $i$ arrangement $-j$ haplotype in the sample; $p_{i}$ and $p_{j}$ are the frequencies of the $i$ arrangement and $j$ haplotype in the sample, respectively.

$$
\begin{aligned}
& D^{\prime}=D / \mid D_{\max }, \text { where } \\
& D_{\max }=\min \left[\left(1-p_{i}\right) p_{j}, p_{i}\left(1-p_{j}\right)\right] \text { if } D>0 \\
& D_{\max }=\max \left[-p_{i} p_{j},-\left(1-p_{i}\right)\left(1-p_{j}\right)\right] \text { if } D<0
\end{aligned}
$$

The statistical significance of the disequilibria was calculated by means of the Fisher's exact test for independence in a $2 \times 2$ contingency table (Sokal and Rohlf, 1995). To diminish any statistical artefacts in the significance that could arise when multiple comparisons are made the following were applied: (i) a sequential Bonferroni test (Rice, 1989) to correct the probabilities when many tests are carried out simultaneously; (ii) only those combinations giving expected numbers $\geq 5$ in all cells were considered for the analysis. Moreover, the least frequent arrangement of each chromosome was omitted to allow for independence among the tests; (iii) only haplotype I was analysed individually, because the results for haplotype II are the same as for haplotype I, but with a change in sign.

The factorial analysis of correspondences (Arenas et al, 1997) was also used to test for any association between haplotypes I and II and the chromosomal arrangements.

\section{Presence of Wolbachia}

To exclude an incompatibility system in D. subobscura, promoted by the presence of Wolbachia, a PCR assay using $16 S$ rDNA Wolbachia-specific primers was carried out following the methodology of García-Martínez et al (1998). In this method, primers were designed according 
to O'Neill et al (1992). These primers amplify a fragment of 869 bp. Two different strains of Sitophilus oryzae containing Wolbachia were used as positive controls. Moreover, two $D$. subobscura regions were used as positive controls for DNA amplifications: the V4-18S rDNA (a 427 bp fragment from the V4 variable region of the $18 \mathrm{~S}$ rDNA) and the ND5 mtDNA (a 984 bp fragment from subunit 5 of NADH dehydrogenase). Six lines (three of haplotype I and three of haplotype II) were used at random from the Calvia population. Since the determination was negative, the presence of Wolbachia in the population was excluded. Only a few lines of $D$. subobscura were used for two reasons: (i) this determination was the second one made on the island of Majorca. The first one was in a different population (Esporles) a few years ago, and was also negative (García Martínez et al, 1998); (ii) we have not detected any kind of cytoplasmic incompatibility in the population, such as embryo mortality or production of all-male progeny in crosses with different strains.

\section{Results}

The cleavage map of the restriction enzymes, based on the physical mtDNA map of D. yakuba (Clary and Wolstenholme, 1985) is shown at the bottom of Figure 1. As can be seen, the NADH and $\mathrm{CO}$ complex genes included most of the polymorphic sites. Table 1 shows the haplotypes found in the Calvia population. As in other populations, haplotypes I and II were the most frequent (95.5\%), with haplotype II being more common than haplotype I. The less common haplotypes accounted for only $4.5 \%$. The relationship between haplotypes I and II and the rarer ones is also indicated in Figure 1, which represents an unrooted phylogenetic tree of the 10 haplotypes of D. subobscura mtDNA. Three haplotypes were derived from haplotype I and five from haplotype II, which were caused by one or two mutations in the mtDNA. In the resolution of this tree, we followed Wagner's parsimony method of the MIX program included in the PHYLIP package (Felsenstein, 1993).

Tajima's D-test was used to detect departures of the mtDNA haplotypic distribution from the neutrality hypothesis. The $D$ value was -1.629 , which was not significant at the $5 \%$ level, but was at $10 \%$, indicating an almost significant excess of rare haplotypes. Table 2 shows the chromosomal arrangement frequencies within haplotypes. Interestingly, the $X$ haplotype had a rare arrangement in the $\mathrm{E}$ chromosome $\left(\mathrm{E}_{1+2+22}\right)$. The $D$ and $D^{\prime}$ values, which detect linkage disequilibrium between the chromosomal arrangements and haplotype I, are presented in Table 3. As can be seen, a significant disequilibrium of haplotype $\mathrm{I}$ and inversion $\mathrm{J}_{\mathrm{ST}}$ was detected. This result was confirmed and complemented by means of a factorial analysis of correspondences. This is a type of analysis based on principal components with $\chi^{2}$ distance, which is appropriate for the representation of the contingency tables and, in our case, a simultaneous representation of the inversions and haplotypes was made in conjunction with the statistical significance of this association. As before, the only significant difference was found with the J chromosome inversions. A statistical association of haplotype I with the $\mathrm{J}_{\mathrm{ST}}$ inversion and haplotype II with $\mathrm{J}_{1}\left(\chi_{(2)}^{2}=10.3, P<0.01\right)$ was found.

\section{Discussion}

The same pattern as reported in previous papers was found with respect to the mtDNA haplotype distribution in the Calvia population (García-Martínez et al, 1998; Castro et al, 1999, and references therein). That is, we detected a high prevalence of haplotypes I and II and a series of less common ones derived from them both. Tajima's $D$ value can help us to understand, at least partly, this result. The negative $D$ obtained is compatible with certain kinds of selection, such as recent directional selection, and also with population bottlenecks. Although purifying selection might also generate a negative $D$ value, it would be expected to be more intense. Therefore, another explanation, based on a population bottleneck seems to be more acceptable. It is possible that the population has not completely reached equilibrium as a consequence of the periodic seasonal bottlenecks (winter and summer), which reduce the effective population size, followed by an expansion and, as a result, an excess of the less common haplotype polymorphism during this expansion. This result, together with previous ones (eg, Fos et al, 1990; Castro et al, 1999), seem to indicate the important effect of random drift on mtDNA in experimental and natural populations. Thus, a possible explanation to account for the coexistent distribution of haplotypes I and II in nature would be that the dynamics of the frequencies of these haplotypes are determined by genetic drift in a population of large effective size. This fact would be reinforced because of the synonymous changes that differentiate the two haplotypes and make them phenotypically equivalent. However, the dynamics are not simple, and other evolutionary forces must be taken into account. For example, in population cages we have also found a clear selective advantage of haplotype II over haplotype I when they are competing together (García-Martínez et al, 1998). This could be a consequence not only of the selection acting directly on the haplotypes, but also of the hitchhiking of mtDNA haplotypes with some nuclear markers. To confirm this possible hitchhiking, a previous cytonuclear association experiment was carried out between nuclear markers (isozymes) and mtDNA haplotypes (Castro et al, 1998, 1999). In that experiment, 13 systems were studied and a disequilibrium associated with Acph-2 was detected, although this could represent a transient disequilibrium established at the moment the samples were taken. In addition, it is possible that the choice of the nuclear markers was not the most appropriate one. The chromosomal polymorphism for inversions of this species is a much better candidate to use in this type of experiment due to its adaptive value (Prevosti et al, 1988; Orengo and Prevosti, 1996). The present study detected a significant association between haplotype I and the $\mathrm{J}_{\mathrm{ST}}$ inversion and between haplotype II and the $\mathrm{J}_{1}$ inversion. This association can hardly be attributed to a random event because of the large number of isofemale lines analysed and the accurate statistics used. Consequently, some kind of epistatic interaction could be considered to explain the equilibrium of haplotypes I and II found in nature. Nevertheless, some resampling should be carried out to confirm this association, since it is possible that it could seasonally disappear and reappear because it could be transient. If this epistatic interaction is real, it could explain the superiority of haplotype II over haplotype I detected by 


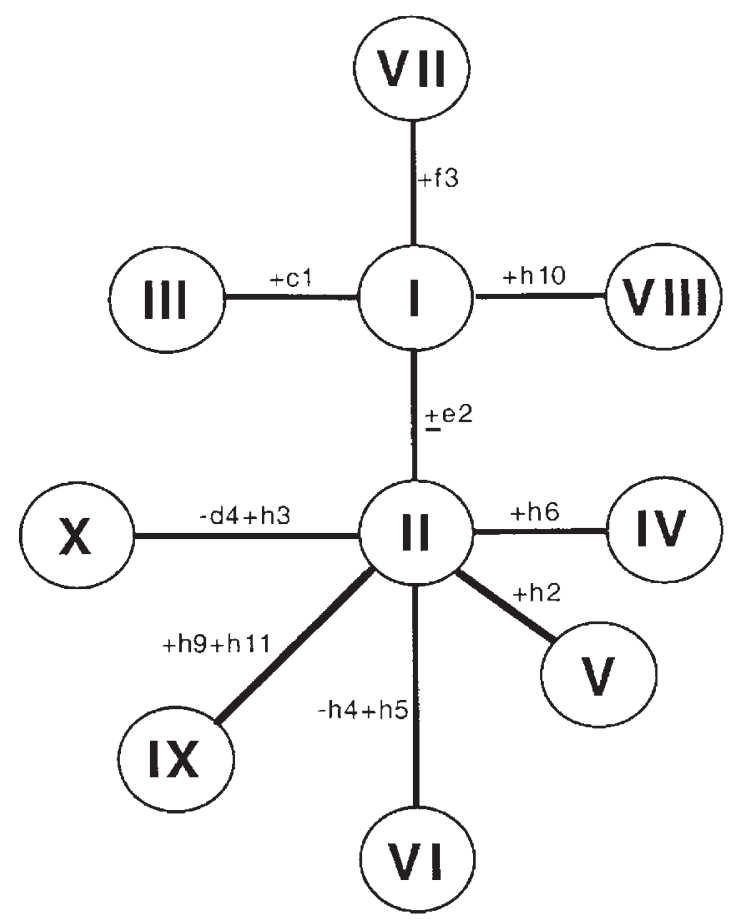

\section{b}

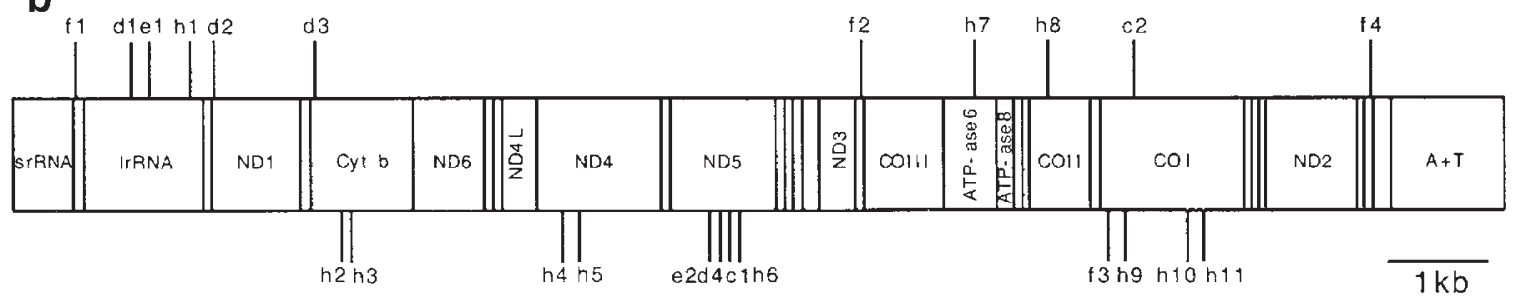

Figure 1 (a) Unrooted phylogenetic tree for the $10 \mathrm{mtDNA}$ haplotypes of the Calvia population of Drosophila subobscura. The haplotypes are connected in a way that minimizes the total number of necessary site changes. The number of mutational steps is indicated on each branch. (b) D. subobscura mtDNA organization based on the genetic map of D. yakuba given by Clary and Wolstenholme (1985). Conserved sites are shown above and polymorphic sites below the map. Abbreviations for the genes are as follows: srRNA and lrRNA, small and large subunits of ribosomal RNA, respectively; ND1-6, subunits of the NADH dehydrogenase complex; Cytb, cytochrome $b$; COI-III, subunits of cytochrome oxidase; $\mathrm{A}+\mathrm{T}$, regulatory noncoding region. Each site is named with a letter, for each one of the restriction endonucleases, followed by a number corresponding to a specific restriction site. (d) EcoRI; (c) EcoRV; (e) HaeIII; (h) HpaII and (f) HindIII.

Table 1 Different haplotypes defined by the polymorphic sites in the sample of Drosophila subobscura from Calvia. Each site is named as in the restriction map. Presence or absence of a given polymorphic site is indicated by a plus (+) or a minus (-) sign, respectively

\begin{tabular}{|c|c|c|c|c|c|c|c|c|c|c|c|c|c|c|}
\hline \multirow[b]{2}{*}{ Haplotype } & \multirow[b]{2}{*}{$\begin{array}{c}E c o R I \\
d 4\end{array}$} & \multirow[b]{2}{*}{$\begin{array}{c}E c o R V \\
c 1\end{array}$} & \multirow[b]{2}{*}{$\begin{array}{c}\text { HaeIII } \\
e 2\end{array}$} & \multirow[b]{2}{*}{$\begin{array}{c}\text { HindIII } \\
f 3\end{array}$} & \multicolumn{8}{|c|}{ HpaII } & \multirow[b]{2}{*}{ No. } & \multirow[b]{2}{*}{$\%$} \\
\hline & & & & & $h 2$ & h3 & $h 4$ & $h 5$ & $h 6$ & h9 & h10 & $h 11$ & & \\
\hline I & + & - & + & - & - & - & + & - & - & - & - & - & 79 & 39.3 \\
\hline II & + & - & - & - & - & - & + & - & - & - & - & - & 113 & 56.2 \\
\hline III & + & + & + & - & - & - & + & - & - & - & - & - & 1 & 0.5 \\
\hline IV & + & - & - & - & - & - & + & - & + & - & - & - & 2 & 1.0 \\
\hline $\mathrm{V}$ & + & - & - & - & + & - & + & - & - & - & - & - & 1 & 0.5 \\
\hline VI & + & - & - & - & - & - & - & + & - & - & - & - & 1 & 0.5 \\
\hline VII & + & - & + & + & - & - & + & - & - & - & - & - & 1 & 0.5 \\
\hline VIII & + & - & + & - & - & - & + & - & - & - & + & - & 1 & 0.5 \\
\hline IX & + & - & - & - & - & - & + & - & - & + & - & + & 1 & 0.5 \\
\hline$x$ & - & - & - & - & - & + & + & - & - & - & - & - & 1 & 0.5 \\
\hline Total & & & & & & & & & & & & & 201 & 100 \\
\hline
\end{tabular}

No. $=$ number of isofemale lines showing a given haplotype. 
Table 2 Chromosomal arrangement frequencies within haplotypes of the Drosophila subobscura population. The sample size is 79 for haplotype I, 113 for haplotype II and nine for haplotypes III to X combined. The $X$ haplotype had a rare arrangement in the E chromosome

\begin{tabular}{|c|c|c|c|}
\hline \multirow[t]{2}{*}{ Arrangement } & \multicolumn{3}{|c|}{ Haplotype } \\
\hline & $I$ & II & $I I I-X$ \\
\hline $\mathrm{A}_{\mathrm{ST}}$ & 0.2911 & 0.3097 & 0.3333 \\
\hline $\mathrm{A}_{1}$ & 0.0380 & 0.0354 & 0.1111 \\
\hline $\mathrm{A}_{2}$ & 0.6709 & 0.6549 & 0.5556 \\
\hline $\mathrm{J}_{\mathrm{ST}}$ & 0.2911 & 0.1150 & 0.2222 \\
\hline $\mathrm{J}_{1}$ & 0.5823 & 0.7699 & 0.4444 \\
\hline $\mathrm{J}_{3+4}$ & 0.1266 & 0.1150 & 0.3333 \\
\hline $\mathrm{U}_{\mathrm{ST}}$ & 0 & 0.0088 & 0 \\
\hline $\mathrm{U}_{2}$ & 0.0380 & 0.0531 & 0.1111 \\
\hline $\mathrm{U}_{1+2}$ & 0.4684 & 0.4690 & 0.4444 \\
\hline $\mathrm{U}_{1+2+8}$ & 0.4557 & 0.4159 & 0.4444 \\
\hline $\mathrm{U}_{2+13}$ & 0.0380 & 0.0531 & 0 \\
\hline $\mathrm{E}_{\mathrm{ST}}$ & 0.1038 & 0.1239 & 0 \\
\hline $\mathrm{E}_{1+2}$ & 0.1429 & 0.2124 & 0.1111 \\
\hline $\mathrm{E}_{1+2+9}$ & 0.1169 & 0.0973 & 0 \\
\hline $\mathrm{E}_{1+2+22}$ & 0 & 0 & 0.1111 \\
\hline $\mathrm{E}_{1+2+9+3}$ & 0.0130 & 0.0088 & 0.1111 \\
\hline $\mathrm{E}_{1+2+9+4}$ & 0.5584 & 0.4513 & 0.6667 \\
\hline $\mathrm{E}_{1+2+9+12}$ & 0.0650 & 0.1062 & 0 \\
\hline $\mathrm{O}_{\mathrm{ST}}$ & 0.0641 & 0.0265 & 0.1111 \\
\hline $\mathrm{O}_{3+4}$ & 0.2051 & 0.2301 & 0.1111 \\
\hline $\mathrm{O}_{3+4+1}$ & 0.0256 & 0.0885 & 0 \\
\hline $\mathrm{O}_{3+4+2}$ & 0.0128 & 0.0088 & 0.1111 \\
\hline $\mathrm{O}_{3+4+7}$ & 0.0513 & 0.0354 & 0 \\
\hline $\mathrm{O}_{3+4+8}$ & 0.4231 & 0.4248 & 0.4444 \\
\hline $\mathrm{O}_{3+4+17}$ & 0.0513 & 0.0088 & 0 \\
\hline $\mathrm{O}_{3+4+22}$ & 0.0128 & 0.0177 & 0.1111 \\
\hline $\mathrm{O}_{3+4+23+6}$ & 0.1538 & 0.1593 & 0.1111 \\
\hline
\end{tabular}

Table 3 Values of $D$ and $D^{\prime}$ between chromosomal arrangements and haplotype I

\begin{tabular}{lrrrr}
\hline & \multicolumn{1}{c}{$D$} & \multicolumn{1}{c}{$D^{\prime}$} & $P$ & $P_{\text {corr }}$ \\
\hline $\mathrm{A}_{\mathrm{ST}}$ & -0.0045 & -0.0362 & 0.8734 & 1.0000 \\
$\mathrm{~A}_{2}$ & 0.0039 & 0.0278 & 0.8774 & 1.0000 \\
$\mathrm{~J}_{\mathrm{ST}}$ & 0.0426 & 0.3864 & 0.0026 & 0.0362 \\
$\mathrm{~J}_{1}$ & -0.0454 & -0.2512 & 0.0069 & 0.0862 \\
$\mathrm{U}_{1+2}$ & -0.0002 & -0.0008 & 1.0000 & 1.0000 \\
$\mathrm{U}_{1+2+8}$ & 0.0096 & 0.0412 & 0.6574 & 0.9999 \\
$\mathrm{E}_{\mathrm{ST}}$ & -0.0048 & -0.1027 & 0.8183 & 1.0000 \\
$\mathrm{E}_{1+2}$ & -0.0168 & -0.2245 & 0.2567 & 0.9617 \\
$\mathrm{E}_{1+2+9}$ & 0.0047 & 0.0752 & 0.8103 & 1.0000 \\
$\mathrm{E}_{1+2+9+4}$ & 0.0258 & 0.1261 & 0.1835 & 0.9122 \\
$\mathrm{E}_{1+2+9+12}$ & -0.0099 & -0.2743 & 0.4399 & 0.9970 \\
$\mathrm{O}_{3+4}$ & -0.0060 & -0.0672 & 0.7252 & 1.0000 \\
$\mathrm{O}_{3+4+8}$ & -0.0004 & -0.0024 & 1.0000 & 1.0000 \\
$\mathrm{O}_{3+4+23+6}$ & -0.0013 & -0.0205 & 1.0000 & 1.0000 \\
\hline
\end{tabular}

$\mathrm{P}_{\text {corr }}=$ Probability corrected by the sequential Bonferroni test.

García-Martínez et al (1998) in population cages, because in this situation, it is possible that some kind of mtDNAinversion interaction (with the same or different arrangements) could be established at the moment the experiments were carried out. Moreover, studies of the fitness components for the haplotypes (I, II and the less common ones) could provide valuable information about their relative selective characteristics.

The association found between the haplotypes and the chromosome J arrangements might be related to the different physiological substrates. The J chromosome contains, among others, the allozyme loci Est-3, Est-7 and Aph (Krimbas, 1993), and the enzymes coded by these loci can be included in a group established by Gillespie and Langley (1974) based on the capacity to make use of different physiological substrates. Differences between the two haplotypes might indicate a differential use of the physiological substrates for each haplotype and differences in the availability of these substrates according to seasonal variation.

Future work should include the study of the fitness components and life-history characters (ie viability and developmental time, longevity, resistance to desiccation, competition, etc) for all the haplotypes, as well as experiments with population cages, but controlling the arrangements, and the variation of the mtDNA at the nucleotide sequence level using additional I, II and rare haplotypes.

\section{Acknowledgements}

This work has been supported by grants PB96-0793 and BOS2000-1000 from the Dirección General de Ensemanza Superior (Ministerio de Educación y Cultura, Spain).

\section{References}

Arenas C, Cuadras CM, Fortiana J (1997). MULTICUA. Paquete no estándar de Análisis Multivariante. Versión 0.77. Publicacions del Departament d'Estadística. Núm. 1.

Ayala FJ, Serra LL, Prevosti A (1989). A grand experiment in evolution: the Drosophila subobscura colonization of the Americas. Genome 31: 246-255.

Castro JA, Picornell A, Ramon MM (1998). Mitochondrial DNA: a tool for populational genetics studies. Int Microbiol 1: 327332.

Castro JA, Ramon MM, Picornell A, Moya A (1999). The genetic structure of Drosophila subobscura populations from the islands of Majorca and Minorca (Balearic Islands, Spain) based on allozymes and mitochondrial DNA. Heredity 83: 271-279.

Clary DO, Wolstenholme DR (1985). The mitochondrial DNA molecule of Drosophila yakuba: nucleotide sequence, gene organization, and genetic code. J Mol Evol 22: 252-271.

Felsenstein J (1993). PHYLIP (Phylogeny Inference Package) version 3.5c. Distributed by the author. Department of Genetics, University of Washington, Seattle.

Fos M, Dominguez MA, Latorre A, Moya A (1990). Mitochondrial DNA evolution in experimental populations of Drosophila subobscura. Proc Natl Acad Sci USA 87: 4198-4201.

García-Martínez J, Castro JA, Ramon MM, Latorre A, Moya A (1998). Mitochondrial DNA haplotype frequencies in natural and experimental populations of Drosophila subobscura. Genetics 149: 1377-1382.

Gerber AS, Loggins R, Kumar S, Dowling TE (2001). Does nonneutral evolution shape observed patterns of DNA variation in animal mitochondrial genomes? Ann Rev Genet 35: 539-566.

Gillespie JH, Langley CH (1974). A general model to account for enzyme variation in natural populations. Genetics 76: 837-884.

Jenkins TM, Babcock CS, Geiser DM, Anderson WW (1996). Cytoplasmic incompatibility and mating preference in Colombian Drosophila pseudoobscura. Genetics 142: 189-194.

Kennedy P, Nachman MW (1998). Deleterious mutations at the mitochondrial ND3 gene in South American marsh rats (Holochilus). Genetics 150: 359-368. 
Kilpatrick ST, Rand DM (1995). Conditional hitchhiking of mitochondrial DNA: frequency shifts of Drosophila melanogaster mtDNA variants depend on nuclear genetic background. Genetics 141: 1113-1124.

Krimbas CB (1993). Drosophila subobscura. Biology, Genetics and Inversion polymorphism. Verlag Dr. Kovac: Hamburg.

Latorre A, Hernández C, Martínez D, Castro JA, Ramon MM, Moya A (1992). Population structure and mitochondrial DNA gene flow in Old World populations of Drosophila subobscura. Heredity 68: 15-24.

Latorre A, Moya A, Ayala FJ (1986). Evolution of mitochondrial DNA in Drosophila subobscura. Proc Natl Acad Sci USA 83: 8649-8653.

Lewontin RC (1964). The interaction of selection and linkage. I. General considerations; heterotic models. Genetics 49: 49-67.

MacRae AF, Anderson WW (1988). Evidence for non-neutrality of mitochondrial DNA haplotypes in Drosophila pseudoobscura. Genetics 120: 485-494.

MacRae AF, Anderson WW (1990). Can mating preferences explain changes in mtDNA haplotype frequencies? Genetics 124: 999-1001.

Moya A, Barrio E, Martinez D, Latorre A, González-Candelas F, Ramon M et al (1993). Molecular characterization and cytonuclear disequilibria of two Drosophila subobscura mitochondrial haplotypes. Genome 36: 890-898.

Nachman MW, Boyer SN, Aquadro CF (1994). Nonneutral evolution at the mitochondrial NADH dehydrogenase subunit 3 gene in mice. Proc Natl Acad Sci USA 91: 6364-6368.

Nachman MW, Brown WM, Stoneking M, Aquadro CF (1996).
Nonneutral mitochondrial DNA variation in humans and chimpanzees. Genetics 142: 953-963.

O'Neill SL, Giordano R, Colbert AME, Karr TL, Robertson HM (1992). 16S rRNA phylogenetic analysis of the bacterial endosymbionts associated with cytoplasmic incompatibility in insects. Proc Natl Acad Sci USA 89: 2699-2702.

Orengo DJ, Prevosti A (1996). Temporal changes in chromosomal polymorphism of Drosophila sobobscura related to climatic changes. Evolution 50: 1346-1350.

Pinto FM, Brehm A, Hernandez M, Larruga JM, Gonzalez AM, Cabrera VM (1997). Population genetic structure and colonization sequence of Drosophila subobscura in the Canaries and Madeira Atlantic islands as inferred by autosomal, sex-linked and mtDNA traits. J Hered 88: 108-114.

Prevosti A, Ribo G, Serra LL, Aguade M, Balaña J, Monclus M et al (1988). Colonization of America by Drosophila subobscura: experiment in natural populations that support the adaptive role of chromosomal-inversion polymorphism. Proc Natl Acad Sci USA 85: 5597-5600.

Rand DM, Kann LM (1998). Mutation and selection at silent and replacement sites in the evolution of animal mitochondrial DNA. Genetica 102-103: 393-407.

Rice WR (1989). Analyzing tables of statistical tests. Evolution 43: 223-225.

Sokal RR, Rohlf FJ (1995). Biometry. Third edition. W.H. Freeman and Co: New York.

Tajima F (1989). Statistical method for testing the neutral mutation hypothesis by DNA polymorphism. Genetics 123: 585-595. 